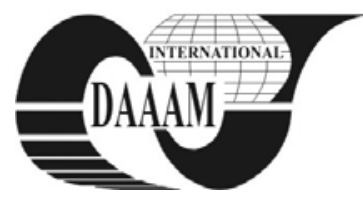

Annals of DAAAM for 2011 \& Proceedings of the 22nd International DAAAM Symposium, Volume 22, No. 1, ISSN 1726-9679 ISBN 978-3-901509-83-4, Editor B. Katalinic, Published by DAAAM International, Vienna, Austria, EU, 2011 Make Harmony between Technology and Nature, and Your Mind will Fly Free as a Bird Annals \& Proceedings of DAAAM International 2011

\title{
ENVIRONMENT FOR 3D COMPUTING
}

\author{
BABINEC, T[omas] \& RICHTER, M[iloslav]
}

\begin{abstract}
This paper deals with new ideas and progress made in the development of a software environment for $3 D$ computations. As the previous versions it is capable of camera intrinsic and extrinsic parameters calibration and $3 D$ point coordinates reconstruction. In addition to that new features have been implemented including linearity and planarity constraints together with lens distortion identification.
\end{abstract}

Key words: photogrammetry, calibration, $3 D$ reconstruction, constraints, optimization

\section{INTRODUCTION}

Computer vision as a relatively young scientific discipline has already proven its importance by significantly contributing to lots of different fields of industry and human life. Although the image processing and all the related tasks are in their essence computationally demanding because of the large amount of data being captured in images, the expansion of the computers' processing power, the development in electronics and mainly the increasing number and quality of digital cameras made it possible to incorporate machine vision into broad number of automation systems. This created a completely new set of engineering tools allowing automation of tasks, which formerly could have been performed by human operators exclusively.

3D computations, reconstructions and modeling form an important part of computer vision. Its main task is to regain the depth (shape) related information about measured objects in the scene via processing sets of obtained $2 \mathrm{D}$ images. The utilization of such algorithms provides exceptionally beneficial ways for measuring and evaluation of scenes' 3D properties. The main advantage in contrast with formerly used methods lies in contactless measuring. In addition to that all the recorded image information can be reprocessed at any time in the future.

Many different programs have been designed in the past to allow computer aided shape evaluation and 3D measurements. Their common disadvantage is that they provide separate algorithms for solving every 3D measurement task. Basic examples of these tasks can be inner and outer camera parameters calibration, lens distortion identification and threedimensional coordinates reconstruction. Independent solution of these so called photogrammetric tasks significantly limits the possibility of cross optimization and error reduction.

This paper deals with a new design concept of an environment for variable $3 \mathrm{D}$ computations. It is aimed at allowing joint and flexible data processing and presents the main advantages of such approach. This work continues in the solution proposed in (Popov et al., 1986) and extends the possibilities and information presented in (Horak et al., 2009).

\section{EARLY CONCEPTS}

There are many different approaches to accomplish the shape evaluation or 3D measurement (Jahne, 1999). There are also many mathematical constraints which can be used for building a scene model and subsequently for computing the unknown parameters. Big number of these is mentioned or can be derived for example from (Sonka et al., 2008) or (Penna \& Patterson, 1986).

In (Popov et al., 1986) the projectivity constraint is used to build scene model by appropriate set of equations linking the parameters divided into following classes: constant, measured and unknown. Also the use of an iterative LSE algorithm was proposed there as an equation set solver.

This groundwork was followed by creation of an environment presented in (Horak et al., 2009). It enabled better utilization of parameter classes, introduced possibility to incorporate additional conditions to the scene model and improved the use of corrections to the measured values.

\section{NEW ENVIRONMENT DESIGN}

Based on the previous work mentioned in section 2 of this paper the environment for 3D computation has been restructured in order to better support the utilization of additional constraints in the scene model and to allow flexible changes to the equation sets. This enables broad option of computational strategies during the estimation of parameter values.

The basic structure of the environment was reduced to 3 modules as shown in Figure 1. The most important one is called MANAGER. It is responsible for photogrammetric data administration. This includes temporary data cache maintenance, automatic compilation of feasible equation sets according to given data dependencies and communication with the other two modules (I/O and CALCULATOR).

The I/O module can have different forms. It serves as a source of photogrammetric data and control commands for the rest of the environment. Therefore, it can be a graphical user interface (GUI), standalone database system or a combination of both.

The third fundamental module is the CALCULATOR. It represents an equation set solver, which is described in more detail in section 3.2.

\subsection{Data types}

There are three main information classes the created environment can work with. These are raw photogrammetric data (point coordinates, camera parameters ...), conditions linking the data and equations defining rigid mathematical formulas between the values of the data.

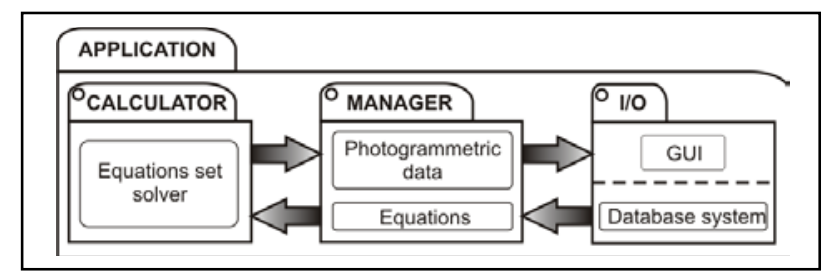

Fig. 1. 3D computing environment scheme 
In order to represent and use the above mentioned kinds of information in an effective way, new hierarchy of data types had to be designed.

The whole scene was divided into specific objects like cameras, views, points, etc. Each of these objects has a defined number of parameters that fully determine their qualities. Every parameter is represented by an instance of class CELL carrying primarily the value, type (unknown, measured, constant) and measure of the deviation of the parameter. For description of an object the cells are grouped into instances of class CLUSTER.

CLUSTER instances also contain conditions given between the objects. Based on these conditions the MANAGER automatically compiles possible equation sets. Instances of the EQUATION class store links to appropriate CLUSTERs and CELLs. Their main purpose is to evaluate the functional formulas $F(\boldsymbol{x}, \boldsymbol{z})$ according to the mathematical constraint they represent. This service is exploited by CALCULATOR module during estimation of parameters' values.

Apart from the projectivity and reprojection error being the fundamental scene model constraint, additional conditions of linearity and planarity of 3D points and lens distortion effect can be defined in the environment. It follows that the 6 basic CLUSTER classes including Camera, View, Picture, 2D \& 3D Points have been extended with 3 additional ones representing lens Distortion and 3D Line \& Plane.

\subsection{Equation set solver}

The equations, from which the scene model is compiled, are nonlinear in general. Algorithms suitable for their solution like gradient based methods, genetic algorithms, differential evolution or modifications of least squares method have an iterative character.

At present iterative adjustment of the parameters by the least squares method is used. Utilization of this algorithm has already been verified in the original work (Popov et al., 1986). It operates with $N$ functions $\boldsymbol{F}(\boldsymbol{x}, \boldsymbol{z})$, which describe photogrammetric relations in a form of $F_{i}(\boldsymbol{x}, \boldsymbol{z})=0$, where $i=1,2, \ldots, N, \boldsymbol{z}=\left[z_{1}, z_{2}, \ldots, z_{P}\right]^{T}$ is a vector of $P$ measured values and $\boldsymbol{x}=\left[x_{1}, x_{2}, \ldots, x_{M}\right]^{T}$ denotes vector of $M$ unknown parameters. The goal of this method is to iteratively optimize unknown parameters in vector $\tilde{\boldsymbol{x}}$ and measured values in $\tilde{\boldsymbol{z}}$ to satisfy equations (1) under the condition (2).

$$
\begin{gathered}
\boldsymbol{F}(\tilde{\boldsymbol{x}}, \tilde{\mathbf{z}})=0 \\
(\tilde{\boldsymbol{z}}-\mathbf{z}) K_{0}^{-1}(\tilde{\boldsymbol{z}}-\mathbf{z})=\text { minimum }
\end{gathered}
$$

$\mathbf{K}_{0}$ is a covariance matrix for the measured values of $\mathbf{z}$.

\begin{tabular}{|c|c|c|c|c|c|c|c|c|}
\hline & $\begin{array}{c}\text { Initial } \\
\text { conditi } \\
\text { ons }\end{array}$ & $\begin{array}{c}\text { Correct } \\
\text { values }\end{array}$ & 1 & 2 & 3 & $\begin{array}{c}\text { Without } \\
\text { lens } \\
\text { distortion } \\
\text { model }\end{array}$ & $\begin{array}{c}\text { Absolute } \\
\text { Error } \Delta\end{array}$ & $\begin{array}{c}\text { Relativ } \\
\text { e error } \\
\delta(\%)\end{array}$ \\
\hline $\begin{array}{c}\mathrm{u}_{0} \\
(\mathrm{pel})\end{array}$ & 400,00 & 400,00 & 400,00 & 400,00 & 400,00 & 346,29 & 53,71 & 13,4 \\
\hline $\begin{array}{c}\mathrm{V}_{0} \\
(\mathrm{pel})\end{array}$ & 300,00 & 300,00 & 300,00 & 300,00 & 300,00 & $-118,31$ & 418,31 & 139,4 \\
\hline $\mathrm{c}(\mathrm{pel})$ & 200,00 & 1000,00 & 323,85 & 468,01 & 1000,00 & 509,83 & 490,17 & 49,0 \\
\hline $\mathrm{w} / \mathrm{h}(-)$ & 0,80 & 1,00 & 1,01 & 1,01 & 1,00 & 0,68 & 0,32 & 31,6 \\
\hline $\mathrm{x}(\mathrm{u})$ & $-2,00$ & 0,00 & 0,16 & 0,16 & 0,00 & 0,02 & $-0,02$ & - \\
\hline $\mathrm{y}(\mathrm{u})$ & 5,00 & 1,00 & 0,77 & 0,77 & 1,00 & 1,06 & $-0,06$ & $-6,0$ \\
\hline $\mathrm{z}(\mathrm{u})$ & $-6,00$ & $-4,00$ & $-2,05$ & $-2,05$ & $-4,00$ & $-4,04$ & 0,04 & $-0,9$ \\
\hline$\omega\left({ }^{\circ}\right)$ & 40,00 & 10,00 & 17,20 & 17,20 & 10,00 & 60,29 & $-50,29$ & $-502,9$ \\
\hline$\varphi\left({ }^{\circ}\right)$ & 20,00 & 0,00 & $-8,04$ & $-8,04$ & 0,00 & $-6,03$ & 6,03 & - \\
\hline$\kappa\left({ }^{\circ}\right)$ & $-20,00$ & 0,00 & 1,60 & 1,60 & 0,00 & 2,07 & $-2,07$ & - \\
\hline $\mathrm{a} 1(-)$ & 6,00 & 1,00 & 6,00 & 0,22 & 1,00 & 0,00 & 0,00 & - \\
\hline $\mathrm{a} 2(-)$ & $-5,00$ & $-0,003$ & $-5,00$ & 0,00 & 0,00 & 0,00 & 0,00 & - \\
\hline
\end{tabular}

Tab. 1. Camera calibration with lens distortion identification. $\left(\left[\mathrm{u}_{0}, \mathrm{v}_{0}\right]=\right.$ center of projection plane, $\mathrm{c}=$ camera constant, camera pose: $[\mathrm{x}, \mathrm{y}, \mathrm{z}]=$ translation vector, $[\omega, \varphi, \kappa]=$ rotation angles, $[\mathrm{a} 1, \mathrm{a} 2]$ = polynomial distortion model parameters.
Application of the Lagrange multipliers method used in (Popov et al., 1986) leads to a computational formulas for the correction vectors $\Delta \boldsymbol{x}$ and $\Delta \boldsymbol{z}$. In each step of the method values of the unknown parameters are updated using the corrections $\Delta \boldsymbol{x}$ according to $\widetilde{\boldsymbol{x}}_{k+1}=\widetilde{\boldsymbol{x}_{k}}+\Delta \boldsymbol{x}$. Iterations should continue until the values in correction vector $\Delta \boldsymbol{x}$ are not sufficiently low. Vector $\Delta \boldsymbol{z}$ then contains corrections to the measured values.

\section{FUNCTION VERIFICATION \& RESULTS}

Table 1 contains results of camera calibration combined with lens distortion identification computed from simulated data with lens effect. The last 3 columns document rather poor results when lens distortion is neglected. The middle part of the Table 1 shows development of the calibration process according to chosen computing strategy. First a good initial guess of projection plane center can be made. Fixing these values and neglecting distortion reduces number of unknowns thus facilitating a rough estimation of external parameters (column “ 1 ”). Next the exterior parameters are frozen, and an estimate of camera distortion is calculated. Finally all camera and distortion parameters are marked as unknown and optimized together in one procedure. Although each step has different equation set they were solved using the same module.

Several different experiments with both artificial and real data involving conditions of planarity and linearity have confirmed usefulness of the described environment concept.

\section{CONCLUSION AND FUTURE WORK}

The presented environment concept has been implemented using $\mathrm{C} / \mathrm{C}++$. Its modular arrangement and hierarchical structure of data types makes it a suitable academic tool for further research in the area of 3D computations. This primarily includes analysis of the influence of additional constraints.

Future work on the environment will involve further extension of implemented scene objects and conditions. There is also a big potential in improving the measurement error propagation as a part of the computing algorithm. Based on this a separate module for intelligent decision making about the course of computational strategies can be designed.

\section{ACKNOWLEDGEMENTS}

This work has been supported in part by Ministry of Education, Youth and Sports of the Czech Republic (Research Intent MSM0021630529 and MSM0021630503), Grant Agency of the Czech Republic (102/09/H081 SYNERGY - Mobile Sensoric Systems and Network) and by Brno University of Technology.

\section{REFERENCES}

Horak K., Richter M., Kalova I.: Application for versatile 3D reconstruction, Proceedings of the $20^{\text {th }}$ International DAAAM Symposium, 2009, Vienna, Austria, ISSN 17269679, ISBN 978-3-901509-70-4, Katalinic, B. (Ed.), pp. 311-312, DAAAM International, Vienna

Jahne B., Haußecker H., Geisler P.: Handbook of Computer Vision and Applications, Volume 1 - 3, Academic Press, ISBN 0-12-379770-5, San Diego, 1999.

Penna, P. \& Patterson, R. (1986). Projective geometry and its applications to computer graphics, Prentice-Hall, ISBN 013-730649-0, USA

Popov P.; Richter, M. \& Solc F. (1995). Exterior orientation of digital images using ccd cameras in close-range photogrammetry, BUT, Brno

Sonka, M.; Hlavac, V. \& Boyle, R. (2008). Image Processing, Analysis, and Machine Vision, Thomson Learning, ISBN 0495-08252-X, USA 